\title{
The role of additional silane coupling agent treatment in oxygen plasma-treated UHMPE fiber/vinylester composites
}

\author{
SUNG IN MOON and JYONGSIK JANG * \\ Department of Chemical Technology, Seoul National University, Shinlimdong, San 56-1, Kwanakgu, \\ Seoul, Korea 151-742
}

Received in final form 8 June 1999

\begin{abstract}
Ultra-high modulus polyethylene (UHMPE) fiber was treated with oxygen plasma and a silane coupling agent in order to improve the interfacial adhesion between the UHMPE fiber and vinylester resin. The oxygen plasma and $\gamma$-methylmethacryloxypropytrimethoxysilane ( $\gamma$-MPS)treated UHMPE fiber/vinylester composites showed a slightly higher interlaminar shear strength than the oxygen plasma-treated UHMPE fiber/vinylester composites. The interfacial adhesion of the oxygen plasma-treated UHMPE fiber/vinylester composites in this study is mainly due to mechanical interlocking between the micropits formed by the oxygen plasma treatment and the vinylester resin. The $\gamma$-MPS molecules adsorbed onto the UHMPE fiber surface neither affected the morphology of the UHMPE fiber surface, nor reduced the extent of mechanical interlocking. The improved interfacial adhesion by the $\gamma$-MPS treatment is due to enhanced wettability and chemical interaction through the chemically adsorbed $\gamma$-MPS molecules, as detected by Fourier-transforminfrared(FT-IR) spectroscopy. The $\gamma$-MPS molecules adsorbed onto the ultra-high molecular weight polyethylene (UHMWPE) plate surface also reduced the aging effect of the oxygen plasma-treated UHMWPE surface.
\end{abstract}

Keywords: UHMPE fiber; oxygen plasma treatment; silane coupling agent; FT-IR; aging effect.

\section{INTRODUCTION}

UHMPE fiber has been widely used for composite materials, due to its excellent tensile modulus, strength, and energy absorption capability [1-12]. However, it has an inert surface and shows poor interfacial adhesion in composite materials. Therefore, surface modification methods for the UHMPE fiber to improve the interfacial adhesion in composite applications have been an important research area [13-26]. Of the various surface treatment methods for the UHMPE fiber,

\footnotetext{
*To whom correspondence should be addressed. E-mail: jsjang@plaza.snu.ac.kr
} 
oxygen plasma treatment is known to be effective for improving the interfacial adhesion in UHMPE fiber composites [13-22].

A great deal of research on the oxygen plasma treatment of the UHMPE fiber has been performed in the case of UHMPE fiber/epoxy composite systems. Interfacial adhesion between the oxygen plasma-treated UHMPE fiber and epoxy resin is improved by both the chemical and the morphological changes of the UHMPE fiber caused by the oxygen plasma. Oxygen plasma treatment oxidizes the inert UHMPE fiber surface and enhances the chemical interaction and wetting of the UHMPE fiber by the epoxy resin. Chemical bonding may exist at the interface and it has been mentioned that this chemical bonding contributes to the interfacial adhesion in oxygen plasma-treated UHMPE fiber/epoxy composites [14, 17, 18]. Oxygen plasma treatment of the UHMPE fiber also introduces micropits on the UHMPE fiber surface, which further improve the interfacial adhesion in UHMPE fiber/epoxy composites through mechanical interlocking between the UHMPE fiber and epoxy resin.

However, when a matrix resin such as unsaturated polyester or vinylester is used, chemical bonding at the interface between the oxygen plasma-treated UHMPE fiber and matrix resin cannot be introduced and the relative contribution of chemical interaction to the interfacial adhesion in UHMPE fiber composites decreases. In this case, oxygen plasma treatment of the UHMPE fiber enhances the wetting of the UHMPE fiber by the matrix resin and enhanced wetting and mechanical interlocking are more important to interfacial adhesion.

Silane coupling agents have been widely applied to glass fiber-reinforced composites in order to improve the interfacial adhesion and mechanical properties [27, 28]. These silane coupling agents transform the glass fiber surface into a surface with good affinity to the matrix resin and thus enhances the interfacial adhesion in glass fiber-reinforced composites. In this study, the oxygen plasma-treated UHMPE fiber was additionally treated with a silane coupling agent in order to improve the interfacial adhesion in UHMPE fiber/vinylester composites.

The role of additional silane treatment in the interfacial adhesion of oxygen plasma-treated UHMPE fiber/vinylester composites was investigated. The effect of silane coupling agent treatment on the morphology of the oxygen plasmatreated UHMPE fiber was studied. The chemical interaction between the oxygen plasma-treated polyethylene and silane coupling agent was investigated using FT-IR spectroscopy. The effect of additional silane treatment on the aging effect of oxygen plasma-treated UHMWPE surface was also determined.

\section{EXPERIMENTAL}

\subsection{Materials}

The UHMPE fiber used was Spectra 900 in plain fabric form from AlliedSignal Co. The matrix resin was XSR-10 vinylester resin from Sewon Chemical Co. (Korea), 
which is a type of vinylester resin modified with carboxyl-terminated acrylonitrile butadiene (CTBN) rubber. Benzoyl peroxide (BPO) was used as a curing initiator and diallylphthalate (DAP) was used as a crosslinking agent. Acetone was used as a solvent for the initiator and as a viscosity reducer.

High-density polyethylene (HDPE) film with a thickness of $15 \mu \mathrm{m}$ from Samsung Chemical Co. (Korea) was used in order to investigate the interface between the oxygen plasma-treated polyethylene surface and the silane coupling agent.

$\gamma$-Methylmethacryloxypropyltrimethoxysilane ( $\gamma$-MPS) from Petrarch Systems Inc. was used as a silane coupling agent for the UHMPE fiber/vinylester composites.

\subsection{Oxygen plasma treatment}

Oxygen plasma treatment of the UHMPE fiber was carried out with plasma treatment apparatus made by Korea Vacuum Co. (Korea). This apparatus is of the parallel electrode type and the sample was placed on a powered electrode to which the RF generator was connected. The diameter of the powered electrode was $35 \mathrm{~cm}$ and the distance between the two electrodes was $8 \mathrm{~cm}$. The oxygen flow rate was fixed at $10 \mathrm{sccm}$ (standard $\mathrm{cm}^{3}$ per $\min$ ) and the plasma output power was adjusted to $100 \mathrm{~W}$.

\section{3. $\gamma$-MPS treatment of the UHMPE fiber}

Distilled water adjusted to $\mathrm{pH} 3.5$ with acetic acid was used for the hydrolysis of $\gamma$-MPS. The concentration of the $\gamma$-MPS was fixed at $0.3 \mathrm{wt} \%$. After prehydrolysis for $1 \mathrm{~h}$, the oxygen plasma-treated UHMPE fiber fabric was dipped into this silane solution for $10 \mathrm{~min}$ and dried for 2 days at room temperature.

\subsection{Prepreg preparation}

The resin bath was made by mixing XSR-10 vinylester resin-BPO-DAP-acetone in the weight ratio of $100: 1.2: 20: 15$. The plasma and silane-treated UHMPE fiber fabric was dipped into this resin bath for $1 \mathrm{~h}$ and dried for 1 day at room temperature.

\subsection{Composite fabrication}

The UHMPE fiber/vinylester composites were fabricated by the open leaky mold method. The curing temperature was $110^{\circ} \mathrm{C}$ and the curing time was $2 \mathrm{~h}$. A curing pressure of 4.1 $\mathrm{MPa}$ was applied after an initial $10 \mathrm{~min}$ and maintained for the rest of the curing period. The UHMPE fiber/vinylester composites consisted of 8 plies of prepregs and the thickness was about $2.7( \pm 0.1) \mathrm{mm}$.

\subsection{Interfacial adhesion measurement}

The relative interfacial adhesion of UHMPE fiber/vinylester composites was measured in terms of the interlaminar shear strength by the three-point short beam 
method according to ASTM D2344. The size of the test specimens was $18 \times$ $10 \times 2.7( \pm 0.1) \mathrm{mm}$. A Lloyd LR-10K universal testing machine was used and the crosshead speed was fixed at $2.0 \mathrm{~mm} / \mathrm{min}$. At least five samples per condition were tested and the results were averaged.

\subsection{SEM analysis}

A JEOL JSM 840A scanning electron microscope (SEM) was used in order to investigate the morphological changes on the UHMPE fiber surface after the oxygen plasma and $\gamma$-MPS treatments. A gold coating was applied to make the samples electrically conductive and the samples were observed at a magnification of 10000 .

\section{8. $\gamma$-MPS treatment of the HDPE film surface}

A methanol $-\mathrm{H}_{2} \mathrm{O}$ (90: 10 by weight) mixture adjusted to $\mathrm{pH} 3.5$ with acetic acid was used as the solvent for the $\gamma$-MPS solution. 1 wt $\% \gamma$-MPS solution was made using this cosolvent and prehydrolyzed for $1 \mathrm{~h}$. Then $0.4 \mathrm{~g}$ of this silane solution was introduced onto the 1 min oxygen plasma-treated HDPE film surface with dimensions of $2 \times 2.5 \mathrm{~cm}$ and the solvent was dried at room temperature. After FT-IR investigation, the silane-treated HDPE film was washed with ethanol to remove the physically adsorbed silane layer. The extent of ethanol washing was controlled by examining the FT-IR spectrum of the ethanol-washed sample after each wash.

\subsection{FT-IR analysis}

A Bomem MB-100 FT-IR spectrometer with a deuterated triglycine sulfate (DTGS) detector was used to obtain the FT-IR spectra. The FT-IR spectrum of neat $\gamma$-MPS was obtained by the transmission technique with $\gamma$-MPS cast on a $\mathrm{KBr}$ pellet. The FT-IR spectrum of the hydrolyzed $\gamma$-MPS was obtained by casting the hydrolyzed $\gamma$-MPS $-\mathrm{H}_{2} \mathrm{O}$ mixture onto a $\mathrm{KBr}$ pellet and drying for $2 \mathrm{~h}$ at $60^{\circ} \mathrm{C}$. The hydrolyzed $\gamma$-MPS $-\mathrm{H}_{2} \mathrm{O}$ mixture was prepared by mixing $\gamma$-MPS and stoichiometrically equivalent $\mathrm{H}_{2} \mathrm{O}$ and hydrolyzed for $4 \mathrm{~h}$ under stirring. A total of 16 scans were added to obtain the spectra of neat and hydrolyzed $\gamma$-MPS and the spectral resolution was fixed at $4 \mathrm{~cm}^{-1}$.

The FT-IR spectra of surface-treated HDPE films were obtained by the transmission technique and the chamber was purged with dry nitrogen to remove the interference of atmospheric $\mathrm{CO}_{2}$ and $\mathrm{H}_{2} \mathrm{O}$. A total of 200 scans were added in order to enhance the signal-to-noise ratio and the resolution was fixed at $4 \mathrm{~cm}^{-1}$.

\subsection{Investigation of the aging effect}

The aging of the oxygen plasma and $\gamma$-MPS-treated UHMWPE plate was investigated using the contact angle method. The UHMWPE plate was prepared by pressing UHMWPE Hizex 240M from Mitsui-Petrochemical using a laboratory hot 
press. The contact angle was measured with a water droplet, using a contact angle goniometer from Rame-Hart. Distilled water was used and the size of the water droplet was fixed at $1 \mu 1$.

\section{RESULTS AND DISCUSSION}

In this study, oxygen plasma treatment and additional $\gamma$-MPS treatment were performed in order to improve the interfacial adhesion of UHMPE fiber/vinylester composites. Figure 1 shows the interlaminar shear strengths (ILSS) of UHMPE fiber/vinylester composites versus the oxygen plasma treatment time. From this figure, it is seen that the $\gamma$-MPS treatment improves the interfacial adhesion of oxygen plasma-treated UHMPE fiber/vinylester composites, although the extent of the improvement is rather small.

The mechanism of the interfacial adhesion improvement in the UHMPE fiber composites caused by the oxygen plasma treatment has been investigated by many researchers $[11-17,20-22]$. Although there are some discrepancies over the most important factor for the interfacial adhesion among these researchers, some important factors are as follows.

Oxygen plasma treatment introduces small micropits on the UHMPE fiber surface by plasma etching. These micropits improve the interfacial adhesion as a result of mechanical interlocking between the resin and these pits [11-15]. Oxygen plasma treatment also introduces oxygen-containing functional groups on the UHMPE fiber surface by plasma surface modification. These oxygen-containing functional groups

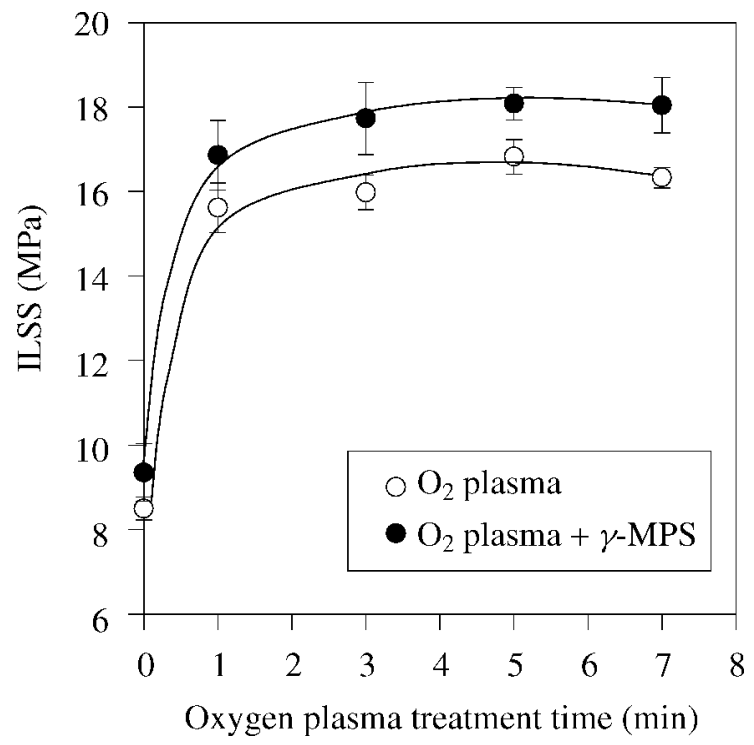

Figure 1. Interlaminar shear strengths (ILSS) of oxygen plasma- or oxygen plasma and $\gamma$-MPStreated UHMPE fiber/vinylester composites versus the oxygen plasma treatment time. 
enhance the chemical interaction between the UHMPE fiber and matrix resin, which further improves the interfacial adhesion of UHMPE fiber composites.

In our system, mechanical interlocking through the micropits is known to be very important in the interfacial adhesion of UHMPE fiber/vinylester composites [11] and many other researchers have also reported the importance of micropits [12-17]. Therefore, in order to improve the interfacial adhesion of the oxygen plasma-treated UHMPE fiber/vinylester composites by $\gamma$-MPS treatment, the $\gamma$-MPS treatment should not reduce the mechanical interlocking through the micropits. Figure 2 shows SEM photographs of the UHMPE fibers after the surface treatments. The introduction of micropits by the oxygen plasma treatment can be seen in Fig. 2a. Figure $2 b$ is a SEM photograph of the oxygen plasma and $\gamma$-MPS-treated UHMPE fiber surface. From this figure, it can be concluded that the $\gamma$-MPS treatment does not change the morphology of the UHMPE fiber surface. This is thought to be due to the low concentration of $\gamma$-MPS solution used in the silane coupling agent treatment. Therefore, the $\gamma$-MPS treatment does not decrease the mechanical interlocking effect in the interfacial adhesion of the UHMPE fiber/vinylester composites.

The improvement in interfacial adhesion in the oxygen plasma-treated UHMPE fiber/vinylester composites by the additional $\gamma$-MPS treatment is mainly due to the improvement in chemical interaction between the UHMPE fiber and vinylester resin through the $\gamma$-MPS treatment.

The $\gamma$-MPS molecules chemically adsorb onto the oxygen plasma-treated UHMPE fiber surface and change the surface properties of the UHMPE fiber. These modified surface properties enhance the chemical interaction between the UHMPE fiber and vinylester resin. There are two types of interface in oxygen plasma- and $\gamma$-MPS-treated UHMPE fiber/vinylester composites. One is the vinylester $/ \gamma$-MPS interface and the other is the $\gamma$-MPS/UHMPE fiber interface. The vinylester/ $\gamma$-MPS interface is almost the same as that in glass fiber-reinforced vinylester composites and has been extensively investigated [27]. The vinylester resin is cured by a radical reaction and it is difficult to induce chemical reaction with the oxygen plasma-treated UHMPE fiber surface, except through the peroxide functional group. However, $\gamma$-MPS contains a methylmethacryl functional group in its structure and this group may participate in the curing reaction of the vinylester resin. It is well known that $\gamma$-MPS on the glass fiber surface participates in the curing reaction of matrix resin in glass fiber-reinforced unsaturated polyester or vinylester composites [27]. Therefore, it is important to confirm the chemical interaction between the $\gamma$-MPS and oxygen plasma-treated UHMPE fiber surface. The possible chemical interactions between the oxygen plasma-treated UHMPE fiber and $\gamma$-MPS are presented schematically in Fig. 3.

In this study, in order to investigate the interaction between the $\gamma$-MPS and the oxygen plasma-treated polyethylene, FT-IR spectroscopy was used. Instead of UHMPE fiber, HDPE film was used for the following reason. When UHMPE fiber is used, it is difficult to control the silane treatment precisely because the silane 

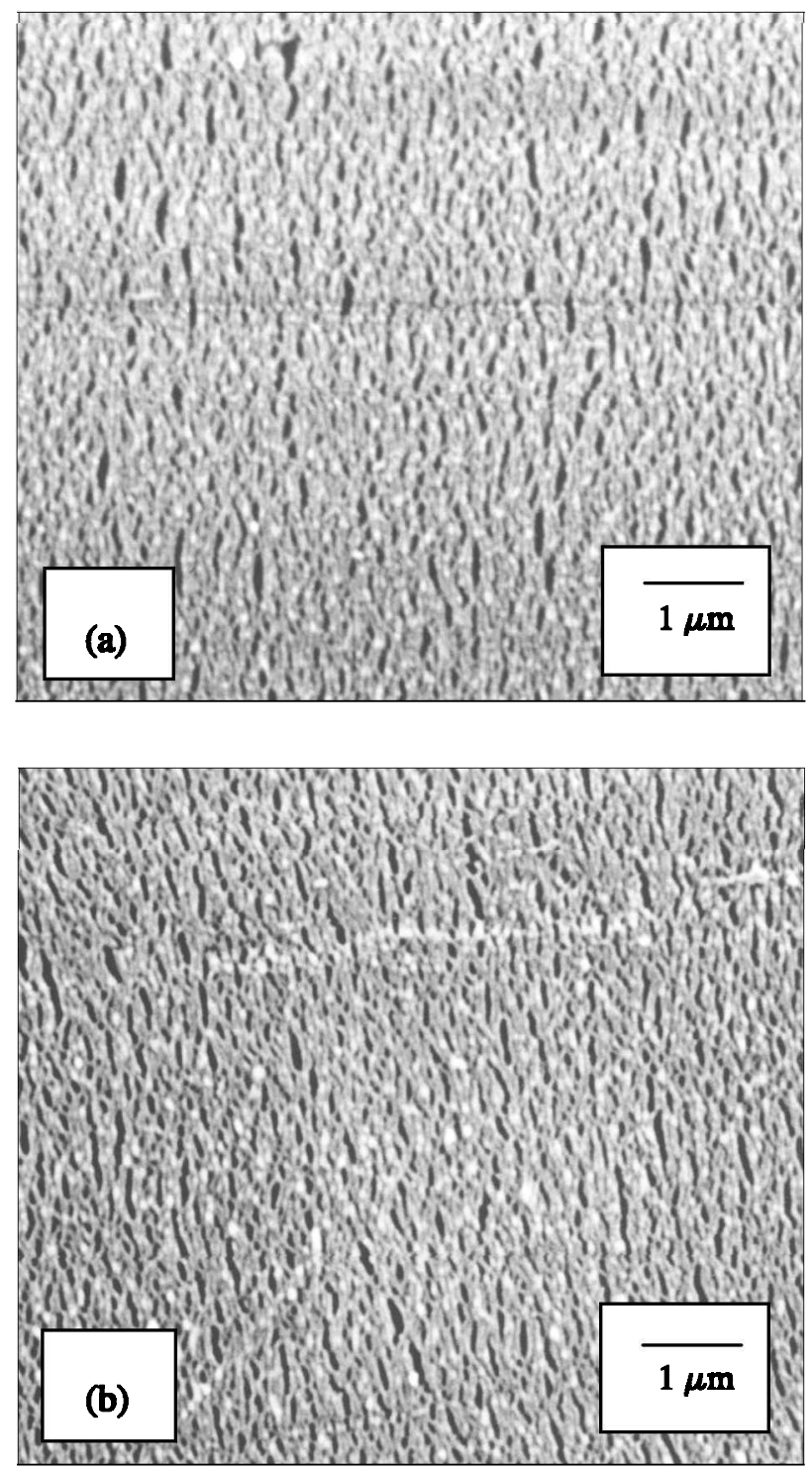

Figure 2. SEM photographs of (a) the 5 min oxygen plasma-treated and (b) the 5 min oxygen plasmaand $\gamma$-MPS-treated UHMPE fiber surface.

solution is absorbed into the UHMPE fiber bundle by the capillary effect and a precise FT-IR spectrum is hard to obtain. The UHMPE fiber used in this study was in plain fabric form and the transmission spectra could not be obtained due to spectral saturation. Other FT-IR techniques such as diffuse reflectance FT-IR spectroscopy were tried, but the enhanced crystalline peaks of the UHMPE fiber interfered with the FT-IR analysis. 
<smiles>C=C(C)C(=O)OCCC[Si](OC)(OC)OC</smiles><smiles>C=C(C)C(=O)OCCC[Si](O)(O)O</smiles><smiles>C=C(C)C(=O)OCCC[Si](O)(O)OC</smiles>

Oxygen plasma-treated UHMPE fiber surface

Figure 3. Schematic diagram of the chemical interactions between the oxygen plasma-treated UHMPE fiber surface and $\gamma$-MPS.

Figure 4 shows the FT-IR transmission spectra of $\gamma$-MPS before and after the hydrolysis reaction. In Fig. $4 \mathrm{a}$, the peak at $1720 \mathrm{~cm}^{-1}$ from $\mathrm{C}=\mathrm{O}$ stretching, the peak at $1089 \mathrm{~cm}^{-1}$ from asymmetric $\mathrm{Si}-\mathrm{O}-\mathrm{CH}_{3}$ stretching, and the peak at $818 \mathrm{~cm}^{-1}$ from symmetric $\mathrm{Si}-\mathrm{O}-\mathrm{CH}_{3}$ stretching can be assigned. After hydrolysis and drying, the peaks from the $\mathrm{Si}-\mathrm{O}-\mathrm{CH}_{3}$ unit decrease and the peaks at 1120 and $1042 \mathrm{~cm}^{-1}$ from $\mathrm{Si}-\mathrm{O}-\mathrm{Si}$ stretching increase as shown in Fig. $4 \mathrm{~b}$. The residual peak near $816 \mathrm{~cm}^{-1}$ in Fig. $4 \mathrm{~b}$ is thought to be due to $\mathrm{Si}-\mathrm{O}$ stretching of free $\mathrm{Si}-\mathrm{OH}$ after the hydrolysis and not from the unhydrolyzed $\mathrm{Si}-\mathrm{O}-\mathrm{CH}_{3}$ because the hydrolysis reaction of $\gamma$-MPS is known to be very fast [27]. 


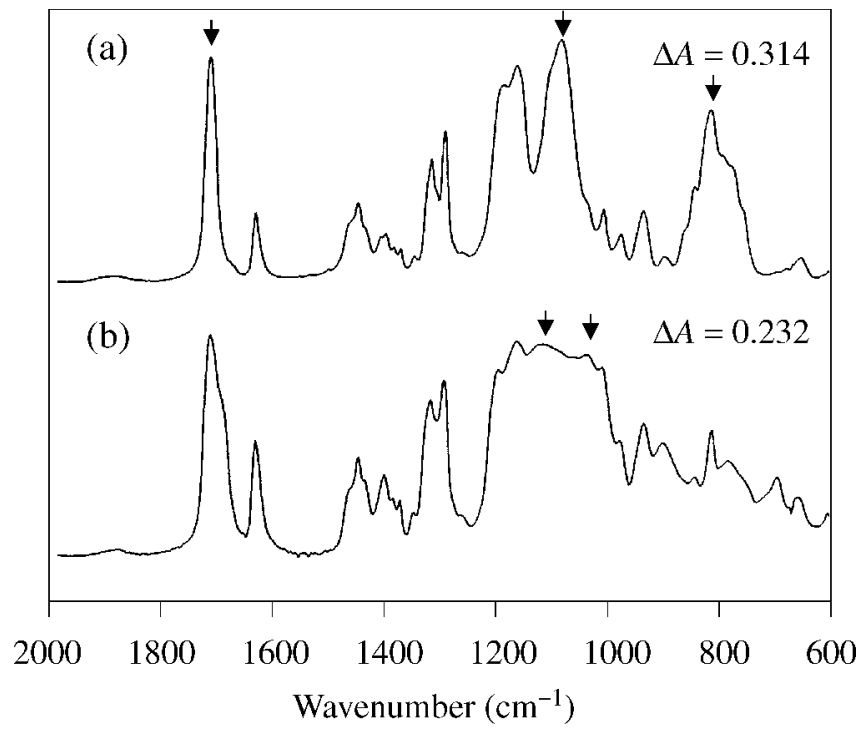

Figure 4. FT-IR transmission spectra of (a) neat $\gamma$-MPS and (b) hydrolyzed and dried $\gamma$-MPS.

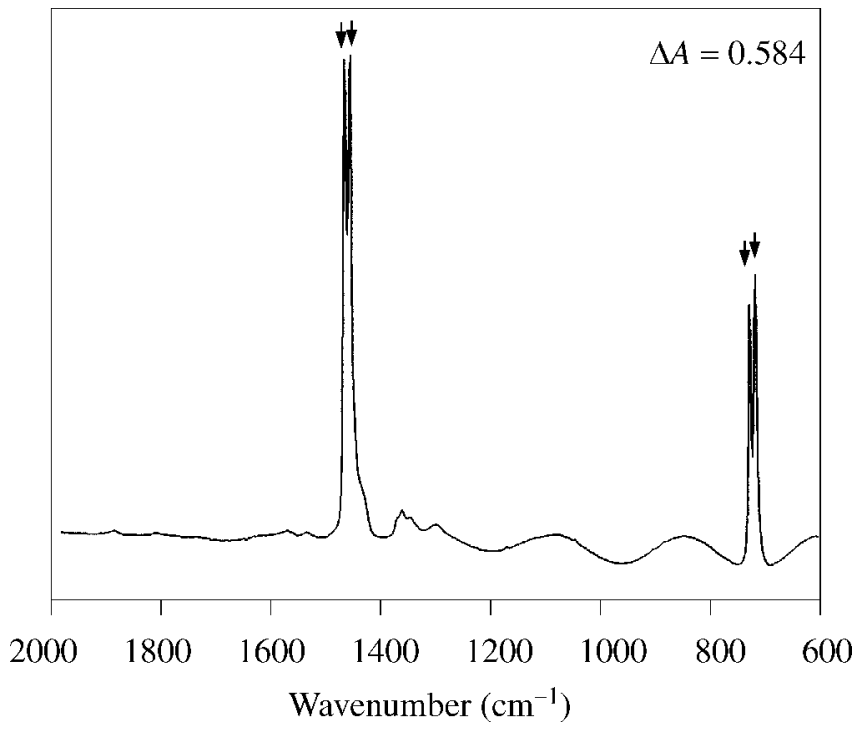

Figure 5. FT-IR transmission spectrum of HDPE film.

The FT-IR transmission spectrum of the HDPE film is shown in Fig. 5. The methylene bending peaks at 1472 and $1463 \mathrm{~cm}^{-1}$ and the methylene rocking peaks at $730,720 \mathrm{~cm}^{-1}$ can be seen.

Figure 6 shows the FT-IR spectra of oxygen plasma-treated HDPE film, additionally $\gamma$-MPS-treated HDPE film, and ethanol-washed HDPE film after the $\gamma$-MPS treatment in the order (a), (b), and (c). The vertical axis of each spectrum is mag- 


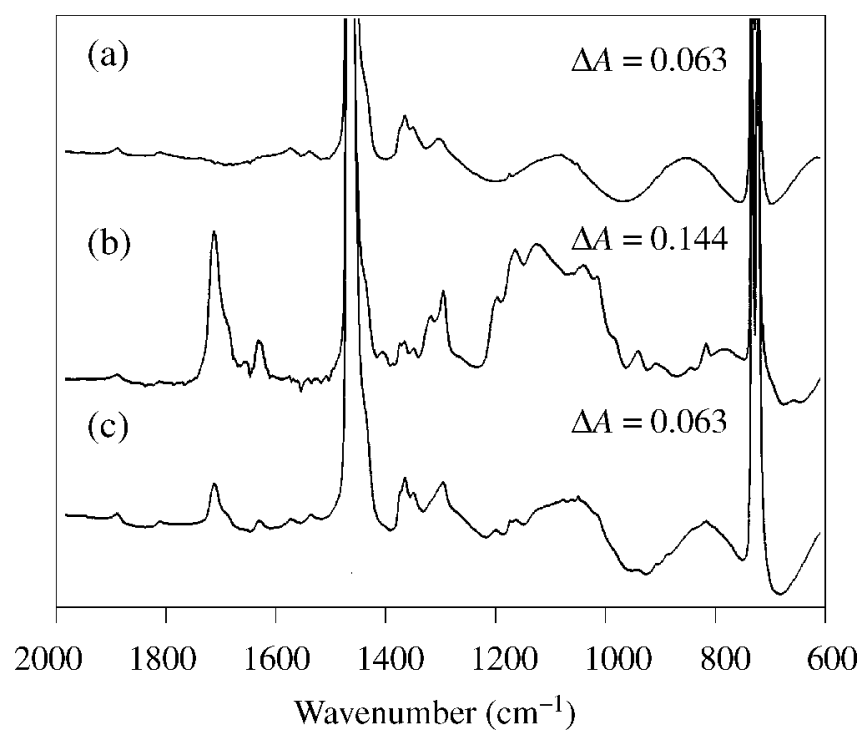

Figure 6. FT-IR transmission spectra of (a) oxygen plasma-treated HDPE film, (b) oxygen plasmaand $\gamma$-MPS-treated HDPE film, and (c) $\gamma$-MPS-treated and ethanol-washed HDPE film.

nified by an arbitrary factor to show the small peaks due to the $\gamma$-MPS molecules more clearly. $\triangle A$ indicates the absorbance difference in the region, excluding the methylene bending and rocking peaks. If $\gamma$-MPS molecules are adsorbed in many molecular layers, the spectrum becomes similar to that of hydrolyzed and dried $\gamma$-MPS shown in Fig. 6b. However, after these physically or weakly chemisorbed silane layers are washed away by ethanol, the spectrum becomes similar to that of HDPE film, although a strong carbonyl stretching peak is seen. This fact can be concluded from comparing the spectra in Figs 6a and 6c. From Fig. 6c, it is difficult to identify the peaks from the $\gamma$-MPS molecules, due to their low intensity. In this case, spectral subtraction can be used to identify the peaks from $\gamma$-MPS molecules on the HDPE film surface.

Figure 7 shows the difference spectra of the spectra shown in Figs $6 \mathrm{~b}$ and $6 \mathrm{c}$, from which the spectrum of the HDPE film in Fig. 6a was subtracted. These difference spectra contain information from the adsorbed $\gamma$-MPS layer. Figure 7 a represents the difference spectrum of the spectrum in Fig. $6 b-6 a$ and Fig. $7 b$ represents the difference spectrum of the spectrum in Fig. $6 c-6 a$. It is very difficult to identify the peaks from chemical bonding of $\gamma$-MPS and oxygen plasma-treated HDPE film directly for the following reasons.

As shown in Fig. 3, $\gamma$-MPS has $\mathrm{Si}-\mathrm{O}-\mathrm{CH}_{3}$ units and these end-groups are transformed into $\mathrm{Si}-\mathrm{OH}$ groups by the hydrolysis. These $\mathrm{Si}-\mathrm{OH}$ (silanol) groups are chemically adsorbed on the HDPE surface through interaction with functional groups such as hydroxyl, carbonyl, and carboxylic acid groups introduced by the oxygen plasma treatment. During the drying process, the $\mathrm{Si}-\mathrm{OH}$ groups condense 


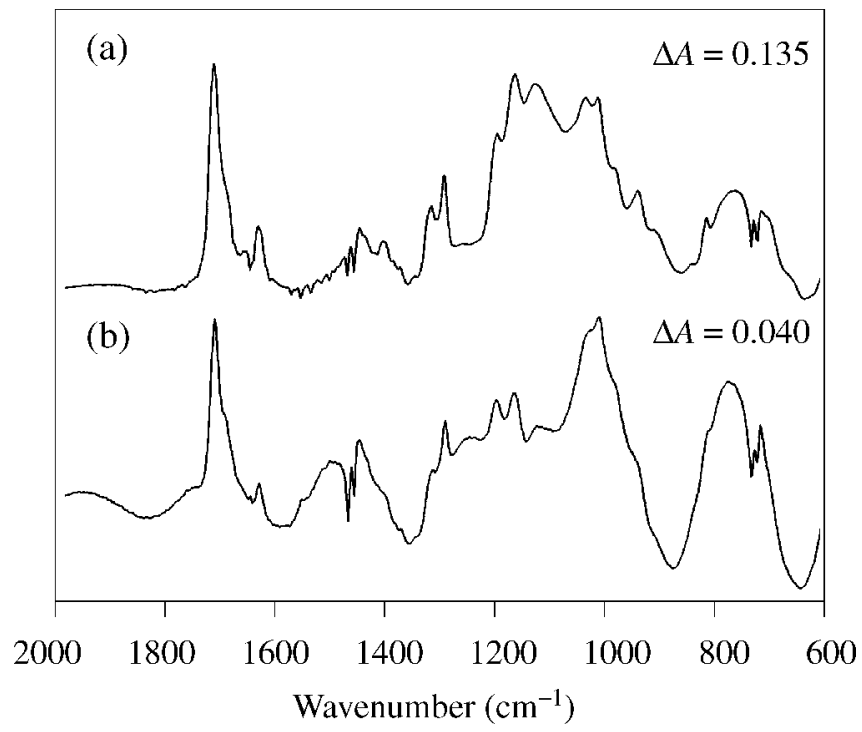

Figure 7. Difference spectra of the surface-treated HDPE film: (a) spectrum in Fig. 6b-6a; (b) spectrum in Fig. 6c-6a.

to $\mathrm{Si}-\mathrm{O}-\mathrm{Si}$ groups and to $\mathrm{Si}-\mathrm{O}-\mathrm{C}$ (on the HDPE surface) groups by reacting with $\mathrm{OH}$ groups on the oxygen plasma-treated HDPE surface.

Therefore, except for the introduction of a $\mathrm{Si}-\mathrm{O}-\mathrm{Si}$ linkage, there are no other changes due to the chemical bonding of $\gamma$-MPS molecules. Unfortunately, the peaks from the $\mathrm{Si}-\mathrm{O}-\mathrm{Si}$ linkage overlap with the peaks from the $\mathrm{Si}-\mathrm{O}-\mathrm{C}$ linkage. Therefore, if the $\gamma$-MPS molecule is adsorbed as many molecular layers, the weak peak from the $\mathrm{Si}-\mathrm{O}-\mathrm{C}$ bond at the interface cannot be identified as the $\mathrm{Si}-\mathrm{O}-\mathrm{Si}$ peaks overlap with the $\mathrm{Si}-\mathrm{O}-\mathrm{C}$ peak.

However, information from the interface can be obtained using the depth profiling technique. If the thickness of the $\gamma$-MPS layer is varied, by comparing the differences from the thick $\gamma$-MPS layer and the thin layer, the state at the interface can be extrapolated.

The spectrum in Fig. 7a is very similar to that of the hydrolyzed and dried $\gamma$-MPS shown in Fig. 4b. However, the spectrum in Fig. $7 \mathrm{~b}$ is different from that in Fig. 4b. The peak at $1129 \mathrm{~cm}^{-1}$ due to $\mathrm{Si}-\mathrm{O}-\mathrm{Si}$ asymmetric stretching is greatly reduced. This means that many of the $\mathrm{Si}-\mathrm{O}-\mathrm{Si}$ bonds have been removed by ethanol washing. The spectrum in Fig. 7b is rather similar to that of neat $\gamma$-MPS in Fig. 4a, although the exact shape is different. This indicates that there are $\mathrm{Si}-\mathrm{O}-\mathrm{C}$ linkages near the $\gamma$-MPS/HDPE interface. It is almost impossible to retain unhydrolyzed $\mathrm{Si}-\mathrm{O}-\mathrm{CH}_{3}$ groups in this experimental condition and this indicates the existence of the $\mathrm{Si}-\mathrm{O}-\mathrm{C}$ (on the HDPE surface) linkage.

Therefore, $\gamma$-MPS molecules are chemically adsorbed onto the oxygen plasmatreated HDPE film and change the surface of HDPE film. The same chemical interaction as that between the $\gamma$-MPS- and oxygen plasma-treated HDPE film can 


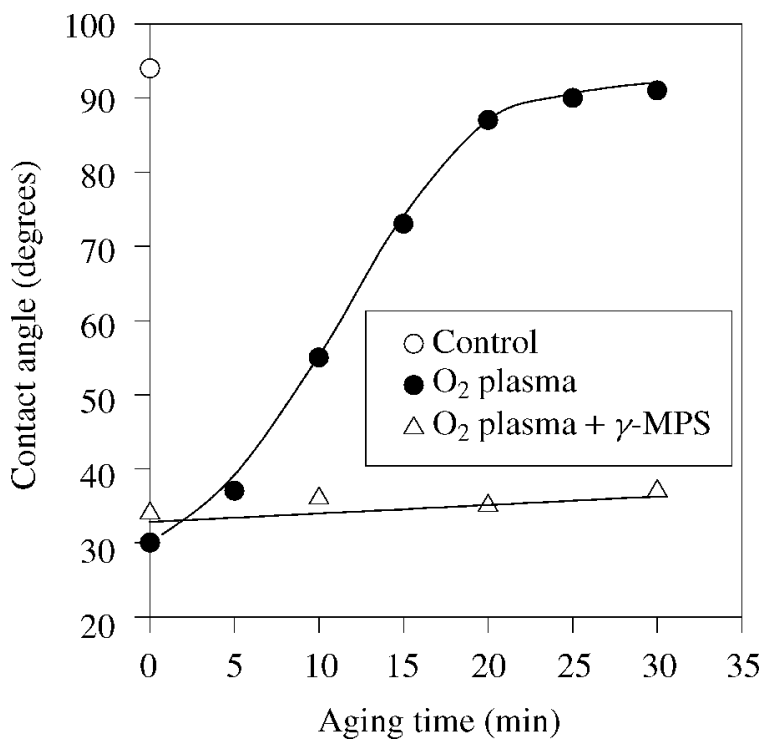

Figure 8. Effect of aging on the contact angle of water on the oxygen plasma- and $\gamma$-MPS-treated UHMWPE surface at $60^{\circ} \mathrm{C}$.

also occur in the case of the UHMPE fiber. This changed surface of the $\gamma$-MPStreated UHMPE fiber enhances the chemical interaction between the UHMPE fiber and vinylester resin.

Another effect of the silane treatment of the oxygen plasma-treated UHMPE fiber is the inhibition of aging of the functional groups. It is well known that the chemical functional groups on the PE surface decrease with the aging time [22, 25]. In this study, aging effects on the oxygen plasma-treated and silane-treated UHMPE fibers were investigated using a molded UHMWPE plate. The degree of aging was measured using the contact angle of water. Figure 8 shows the change in water contact angle on the 5 min oxygen plasma- and silane-treated UHMWPE plate with the aging time. The aging temperature was fixed at $60^{\circ} \mathrm{C}$. This aging experiment was carried out with a molded UHMWPE plate and because of the low crystallinity the speed of the aging of the oxygen plasma-treated UHMWPE plate is rather fast.

From this figure, it can be concluded that $\gamma$-MPS greatly reduces the aging of the functional groups. The $\gamma$-MPS-treated surface is a crosslinked $\gamma$-MPS surface rather than an oxygen plasma-treated surface. The $\gamma$-MPS treatment increases the total number of functional groups on the UHMWPE plate surface and both the bulkiness and the chemical interaction of the $\gamma$-MPS molecules with the UHMWPE surface inhibit the aging of the functional group introduced on the UHMWPE surface by the oxygen plasma treatment. The $\gamma$-MPS molecules condense and are adsorbed on the oxygen plasma-treated UHMWPE surface. It is difficult for the bulky $\gamma$-MPS molecules or condensates to migrate into the bulk of the UHMWPE plate. The chemical interaction between the $\mathrm{Si}-\mathrm{OH}$ groups and the functional 
groups on the UHMWPE surface inhibits the migration of the functional groups introduced on the UHMWPE surface by the oxygen plasma treatment. The same interaction can also occur at the $\gamma$-MPS/oxygen plasma-treated UHMPE fiber interface.

\section{CONCLUSIONS}

The interfacial adhesion in oxygen plasma- and $\gamma$-MPS-treated UHMPE fiber/ vinylester composites was investigated and the following conclusions can be drawn.

The interfacial adhesion in oxygen plasma-treated UHMPE fiber/vinylester composites is greatly affected by the mechanical interlocking between the micropits introduced by the oxygen plasma treatment and the vinylester resin. Additional $\gamma$-MPS treatment slightly improves the interfacial adhesion in the UHMPE fiber/vinylester composites in comparison with the oxygen plasma-treated UHMPE fiber composites. The additional $\gamma$-MPS treatment does not decrease the effectiveness of mechanical interlocking through the micropits. This improved interfacial adhesion by the $\gamma$-MPS treatment is due to the enhanced wetting and chemical interaction of the $\gamma$-MPS-treated UHMPE fiber surface with the vinylester resin.

The $\gamma$-MPS molecules were found to be chemically adsorbed onto the oxygen plasma-treated polyethylene surface by FT-IR analysis. The additional $\gamma$-MPS treatment also decreased the aging effect of the oxygen plasma-treated UHMWPE surface.

\section{REFERENCES}

1. P. Smith and P. J. Lemstra, J. Mater. Sci. 15, 505 (1980).

2. A. Ciferri and I. M. Ward (Eds), Ultra-high Modulus Polymers. Applied Science Publishers, London (1977).

3. P. J. Barham and A. Keller, J. Mater. Sci. 20, 2281 (1985).

4. G. A. George, in: Polymer Surfaces and Interfaces 2, W. J. Feast, H. S. Munro and R. W. Richards (Eds), pp. 161-201. John Wiley, New York (1993).

5. D. C. Prevorsek, in: Reference Book for Composite Technology, S. M. Lee (Ed.), pp. 167-174. Technomic Publishing, Lancaster, PA (1989).

6. D. F. Adams, R. S. Zimmerman and H. Chang, Proc. 30th Int. SAMPE Symp., p. 280 (1985).

7. B. Tissington, G. Pollard and I. M. Ward, Composites Sci. Technol. 44, 197 (1992).

8. D. W. Woods, P. J. Hine and I. M. Ward, Composites Sci. Technol. 52, 397 (1994).

9. B. Z. Jang, L. C. Chen, L. R. Hwang, J. E. Hawkes and R. H. Zee, Polym. Composites 11, 144 (1990).

10. Sung-in Moon and Jyongsik Jang, Polym. Composites 16, 325 (1995).

11. Sung-in Moon and Jyongsik Jang, Composites Sci. Technol. 57, 197 (1997).

12. I. M. Ward and N. H. Ladizesky, Pure Appl. Chem. 57, 1641 (1985).

13. N. H. Ladizesky and I. M. Ward, Composites Sci. Technol. 26, 129 (1986).

14. H. X. Nguyen, G. Riahi, G. Wood and A. Poursartip, Proc. 33rd Int. SAMPE Symp., Anaheim, CA, p. 1721 (1988).

15. N. H. Ladizesky and I. M. Ward, J. Mater. Sci. 24, 3763 (1989). 
16. B. Tissington, G. Pollard and I. M. Ward, J. Mater. Sci. 26, 82 (1991).

17. M. Nardin and I. M. Ward, Mater. Sci. Technol. 3, 814 (1987).

18. J. R. Brown, P. J. C. Chappell and Z. Mathys, J. Mater. Sci. 27, 3167 (1992).

19. J. R. Brown, P. J. C. Chappell and Z. Mathys, J. Mater. Sci. 27, 6475 (1992).

20. H. Rostami, B. Iskandarani and I. Kamel, Polym. Composites 13, 207 (1992).

21. S. Gao and Y. Zeng, J. Appl. Polym. Sci. 47, 2065 (1993).

22. S. Gao and Y. Zeng, J. Appl. Polym. Sci. 47, 2093 (1993).

23. A. Taboudoucht, R. Opalko and H. Ishida, Polym. Composites 13, 81 (1992).

24. Z. F. Li, A. N. Netravali and W. Sachse, J. Mater. Sci. 27, 4625 (1992).

25. C. D. Volpe, L. Fambri, R. Fenner, C. Migliarese and A. Pegoretti, J. Mater. Sci. 29, 3919(1994).

26. K. Takagi, H. Fujimatsu, H. Usami and S. Ogasawara, J. Adhesion Sci. Technol. 10, 869 (1996).

27. E. P. Plueddemann, Silane Coupling Agents. Plenum Press, New York (1982).

28. K. L. Mittal (Ed.), Silanes and Other Coupling Agents. VSP, Utrecht, The Netherlands (1992). 